der, years of experience, practice setting and number of pregnant patients with $\mathrm{RD}$ seen per month), their opinion on pregnancy in RD patients (pre-conception status of RD and counseling, remission length before allowing pregnancy) and compatible RD drugs with pregnancy and lactation. Qualitative variables were analyzed using Chi-square or Fisher's exact tests, while quantitative variables were analyzed using Wilcoxon or Student $t$ tests. A total score of answers per physician in accordance with EULAR/BSR guidelines was calculated. Univariate and multivariate analysis assessed the variables significantly associated with this score. $\mathrm{P}$ value $<0.05$ indicated statistical significance.

Results: Thirty-nine $(90.7 \%)$ rheumatologists participated in this survey. They had $22.2 \pm 11.6$ years of practice. Ninety-four $(41.4 \%)$ obstetricians participated in this survey. They had $26.0 \pm 12.8$ years of practice. Figure 1 displays the percentage of physicians who consider the selected RD medications detrimental to/not compatible with fertility, pregnancy and breastfeeding.

Average score of answers in accordance with EULAR/BSR guidelines was $0.654 \pm 0.154$ and results were significantly different between specialties $(p<0.001)$ : rheumatologists scored between 0.41 and 1 , with an average of $0.777 \pm 0.152$ and obstetricians scored between 0.36 and 1 with an average of $0.603 \pm 0.124$. Multivariable analysis showed that the scores reflecting adherence to recommendations were significantly associated with type of specialty (rheumatology $\mathrm{v} / \mathrm{s}$ obstetrics) and number of pregnant patients with RD seen per month.

Conclusions: Advices given to RD patients who plan on becoming pregnant in Lebanon vary largely, especially among the physicians' specialty and experience in RD, which highlights the urgency of disseminating the EULAR/BSR guidelines among rheumatologists and obstetricians alike.

References:

[1] Flint J et al, Rheumatology 2016.

[2] Götestam Skorpen C et al, Ann Rheum Dis 2016.

[3] Panchal S et al, Rheum Int 2013.

[4] Ruiz-Irastorza G and Khamashta MA, ACR 2014.

Acknowledgements: Sponsor: Newbridge Pharmaceuticals. MW support: KBPBiomak.

Disclosure of Interest: None declared

DOI: 10.1136/annrheumdis-2017-eular.3430

\section{AB0318 CLINICAL AND SEROLOGIC CHARACTERISTICS OF A COHORT OF ECUADORIAN MEN WITH RHEUMATOID ARTHRITIS}

\section{G. Maldonado ${ }^{1}$, C. Ferro ${ }^{2}$, R. Guerrero ${ }^{3}$, C. Paredes ${ }^{3}$, C. Rios ${ }^{4},{ }^{1}$ Universidad} Espiritu Santo, Universidad Espíritu Santo; ${ }^{2}$ Universidad Católica Santiago de Guayaquil; ${ }^{3}$ Universidad Espíritu Santo; ${ }^{4}$ Centro de Reumatología y

Rehabilitación, Guayaquil, Ecuador

Background: Rheumatoid arthritis (RA) is an inflammatory and disabling disease whose expression and clinical course is influenced by gender. In developed countries the prevalence of RA is $0.5-1 \%$, with a ratio $3: 1$ female:male. Comparative gender studies have shown that women have greater functional disability, disease activity and pain than men1.

Objectives: The purpose of this study is to describe and compare the clinical and serological characteristics of a cohort of men with RA.

Methods: A cross-sectional study was conducted in patients with established diagnosis of RA (American College of Rheumatology criteria 1988) from the Luis Vernaza Hospital and a private rheumatology center (CERER). The data included clinical manifestations, demographic data, comorbidities, habits, treatment, activity index DAS28-CRP, HAQ-DI disability index and the PHQ-9 questionnaire. The statistical program SPSS V. 22 was used to analyze the data and calculate frequencies, percentages, means, ranges, Spearman correlation and ANOVA coefficient. Statistical significance used was 0.05 , with a $95 \%$ reliability.

Results: A cohort of 50 men was compared with a control group of 50 women. Most men worked while women stayed at home $(72 \%$ and $66 \%$, respectively, $\mathrm{p}=0.000$ ). Tobacco and alcohol were more common in men ( $34 \%$ vs $8 \% \mathrm{p}=0.001$ and $38 \%$ vs. $0 \% \mathrm{p}=0.000$ respectively). The delay in the first visit to the specialist was shorter in men than in women (24 vs 32 months) and they had a longer follow-up (55 vs 41 months). In clinical manifestations, women had greater fatigue ( $60 \%$ vs $30 \% p=0.003)$, weight loss $(44 \%$ vs $20 \% p=0.010)$, loss of appetite ( $54 \%$ vs $12 \% \mathrm{p}=0.000)$ and depression $(60 \%$ vs $22 \% \mathrm{p}=0.000)$. There was no significant difference in comorbidities and treatment between the groups. Men had lower disease activity: VSG ( 23 vs $47 p=0.046)$, DAS 28 (2.5 vs $3.4 p=0.011$ ), HAQ DI (0.37 vs $1.12 p=0.000)$, functional disability $(12 \%$ vs $36 \% p=0.005)$, severe disability ( $2 \%$ vs $22 \% p=0.002$ ), painful joints ( 2 vs $8 p=0.000$ ), swollen joints (2 vs $6 p=0.000$ ) and VAS of doctor (2 vs $3 p=0.047$ ).

Conclusions: This is the first cohort study of men with RA in Ecuador. As shown in other international studies2-3, our results confirm that men have a less aggressive disease.

\section{References:}

[1] Lesuis N, Befrits R, Nyberg F, van Vollenhoven RF. Gender and the treatment of immune-mediated chronic inflammatory diseases: rheumatoid arthritis, inflammatory bowel disease and psoriasis: an observational study. BMC Med. BioMed Central Ltd;2012;10(1):82.

[2] Sokka T, Toloza S, Cutolo M, Kautuainen H, Makinen H. Women, men, and rheumatoid arthritis: analyses of disease activity, disease characteristics, and treatments in the QUEST-RA Study. Arthritis Res Ther. 2009;11(1):1-12.

[3] Hallert E, Thyberg I, Hass U, Skargren E, Skogh T. Comparison between women and men with recent onset rheumatoid arthritis of disease activity and functional ability over two years (the TIRA project). Ann Rheum Dis. 2003;62(7):667-70.

Disclosure of Interest: None declared

DOI: 10.1136/annrheumdis-2017-eular.1409

\section{AB0319 COMORBIDITIES IN ECUADORIAN PATIENTS WITH RHEUMATOID ARTHRITIS}

G. Maldonado ${ }^{1}$, C. Rios ${ }^{2}$, C. Ferro ${ }^{3}$, R. Guerrero ${ }^{4}$, C. Paredes ${ }^{4}$, M. Moreno ${ }^{5}$, C. Vallejo ${ }^{6} .{ }^{1}$ Universidad Espiritu Santo, Universidad Espíritu Santo; ${ }^{2}$ Centro de Reumatología y Rehabilitación; ${ }^{3}$ Universidad Católica Santiago de Guayaquil; ${ }^{4}$ Universidad Espíritu Santo; ${ }^{5}$ Hospital Luis Vernaza; ${ }^{6}$ Sociedad Ecuatoriana de Reumatología, Guayaquil, Ecuador

Background: Rheumatoid arthritis (RA) is associated with several comorbidities that reduce life expectancy. These comorbidities may be due to the rheumatic process itself, treatment or be independent, and include: anemia, osteoporosis, fractures, infections, gastric ulcers, myocardial infarction, depression, lymphoma and other malignancies.

Objectives: The aim of this study is to determine the comorbidities in patients with an establish diagnosis of rheumatoid arthritis.

Methods: A cross-sectional descriptive study was conducted in patients with an established diagnosis of RA according to the ACR 1987 criteria, from public and private rheumatology clinics in Guayaquil, Ecuador. The database included; demographics, comorbidities, habits, treatments, laboratory exams, number of swollen and tender joints, Visual Analog Scale (VAS), activity and disability index (DAS28 and HAQ-DI) and PHQ-9 for depression. The statistical program SPSS V. 22 was used to analyze the data and calculate frequencies, percentages, means, ranges, Spearman correlation and ANOVA coefficient. Statistical significance used was 0.05 , with a $95 \%$ reliability.

Results: 402 patients with a mean age of 50 years were included, 87.8\% (353) women and $12.2 \%$ (49) men. $93.8 \%$ (377) were mestizos, 3.5\% (14) White, $1.5 \%$ (6) Indigenous and $1.2 \%$ (5) Afro-Ecuadorians. As for the marital status $55.5 \%$ (223) were married, $15.4 \%$ (62) cohabiting, $12.4 \%$ (50) divorced, $8.5 \%$ (34) singles and $8.2 \%$ (33) widowers. 9.7\% (39) of the patients smoked, $7.5 \%$ (30) ingested alcohol and $0.5 \%$ (2) drugs. The average age of onset of the disease was 41 years, with a median time from onset of symptoms and the first rheumatology visit of 28.7 months, median follow-up time of 51.8 months and average disease duration of 11 years. For comorbidities, $54.8 \%(147 / 268)$ had dyslipidemia, 28.4\% (114) gastric disease, 23.6\% (95) hypertension, 20.1\% (81) obesity, $19.6 \%(79)$ depression, $15.2 \%(61)$ thyroid disease, $12.4 \%(50)$ of the sexual involvement, $10.7 \%(43)$ allergies, $10.4 \%$ (32/307) hypertransaminasemia, $9.3 \%(21 / 225)$ anemia, and $6.7 \%$ (27) diabetes mellitus. $32.3 \%$ (114) of women have had at least one abortion. The PHQ-9 medium was 5.3; according to this $11.2 \%$ (45) corresponded to mild depression, $4.5 \%$ (18) moderate depression, $3.2 \%$ (13) moderately severe depression and $0.7 \%$ (3) severe depression. As for the other indicators of disease activity, the average for the number of painful joints was $5(0-28)$, swollen joints $4(0-26)$, patient VAS $3.9(0-10)$ and doctor VAS 3.4 (0-10). In laboratory data, 67.4\% (215/319) had positive ESR, 64.8\% (149/230) PCR, 90.5\% (182/201) anti CCP positive, and 94.4\% (286/303) RF.

Conclusions: This is the first study of comorbidities in patients with RA in Ecuador. Patients with RA have a high prevalence of comorbidities and risk factors, which is why physicians should be prepared to prevent them and offer an early treatment.

Disclosure of Interest: None declared

DOI: 10.1136/annrheumdis-2017-eular.1408

\section{AB0320 RHEUMATOID ARTHRITIS IN BRAZIL - THE "REAL" STUDY: A NATIONWIDE PROSPECTIVE STUDY}

G. Castelar-Pinheiro ${ }^{1}$, A.B. Vargas-Santos ${ }^{1}$, C. Albuquerque ${ }^{2}$, R. Amorim ${ }^{1}$, R. Giorgi ${ }^{3}$, S. Radominski ${ }^{4}$, I. Pereira ${ }^{5}$, M.F. Guimarães ${ }^{6}$, M. Bértolo ${ }^{7}$, P. Louzada Jr. ${ }^{8}$, M.F. Cunha ${ }^{9}$, K. Bonfiglioli ${ }^{10}$, C. Brenol ${ }^{11}$, L.M.H. Mota ${ }^{2}$ ${ }^{1}$ Internal Medicine, Rheumatology Unit, Universidade do Estado do Rio de Janeiro, Rio de Janeiro; ${ }^{2}$ Universidade de Brasília, Brasília; ${ }^{3}$ Instituto de Assistência Médica ao Servidor Público Estadual, São Paulo; ${ }^{4}$ Universidade Federal do Paraná, Curitiba; ${ }^{5}$ Universidade Federal de Santa Catarina, Florianópolis; ${ }^{6}$ Universidade Federal de Minas Gerais, Belo Horizonte; ${ }^{7}$ Universidade Estadual de Campinas, Campinas; ${ }^{8}$ Universidade de São Paulo Ribeirão Preto, Ribeirão Preto; ${ }^{9}$ Universidade Federal do Pará, Belém:

${ }^{10}$ Universidade de São Paulo, São Paulo; ${ }^{11}$ Universidade Federal do Rio Grande do Sul, Porto Alegre, Brazil

Background: Early diagnosis and immediate treatment are critical in achieving optimal outcomes in rheumatoid arthritis (RA). However, anecdotal evidence suggests that in Brazil there is still a significant delay in patient referral, RA diagnosis and DMARD initiation. The lack of national data hinders effective advocacy for proper public health policies, which led the RA commission of the Brazilian Society of Rheumatology to create a nationally-representative multicenter RA cohort to provide the necessary data.

Objectives: To describe the development process and baseline characteristics of a large, nationally-representative multicenter cohort of RA patients in Brazil. 